
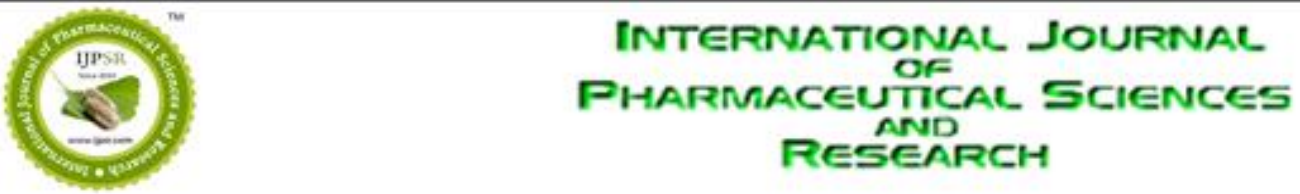

Received on 15 December, 2012; received in revised form, 26 January, 2013; accepted, 22 March, 2013

\title{
ROLE OF THE PHARMACEUTICAL CARE IN THE MANAGEMENT OF PATIENTS WITH TYPE 2 DIABETES MELLITUS
}

Taha O. Mahwi*1 and Kawa A. Obied ${ }^{* 2}$

Department of Internal Medicine, School of Medicine, Sulaimani University, Kurdistan-Iraq

Department of Clinical Pharmacy, School of Pharmacy, Sulaimani University, Kurdistan-Iraq

Keywords:

Diabetes, Pharmaceutical care, Glycemic control, Clinical Pharmacist, Glycosylated haemoglobin, Sulaimani

\section{Correspondence to Author:}

Kawa A. Obeid

Department of Clinical Pharmacy, School of Pharmacy, Sulaimani University, Kurdistan-Iraq

E-mail: kawa.ahmad@gmail.com
Objective: The study was designed to evaluate the efficiency of pharmaceutical care on the control of clinical parameters, such as fasting glycaemia and glycosylated haemoglobin also to assess drug therapy problems in patients with type 2 diabetes mellitus. Setting: the study was conducted at Diabetic Center in Sulaimany/Iraq, during 18 September 2010 -20 January 2011.

Methods: A prospective and randomized control trial study was conducted with 130 type 2 diabetes patients with glycosylated haemoglobin of higher than $7.0 \%$, they were divided into two groups: (i) pharmaceutical care (intervention) group $(n=65)$, and (ii) the control group $(n=65)$. They were monitored for 3 consecutive visits. Patients in the control group received usual medical care, but patients in the intervention group received both standard medical care and pharmaceutical care.

Results: At the end of the study, a statistically significant reduction was observed in the glycemic levels of patients in the intervention group whilst a small reduction, which is statistically not significant, was detected in the control group. Furthermore, the follow-up of the intervention group by a pharmacist contributed to the resolution of 108 drug therapy problems identified.

Conclusion: pharmaceutical care process provided by pharmacist to patients with type 2 diabetes mellitus could yield measurable improvements in the glycemic control, resolution of drug therapy problems and improvements in the compliance toward antidiabetic medication.
INTRODUCTION: Diabetes mellitus is a chronic disease that is associated with high morbidity and mortality from its complications.

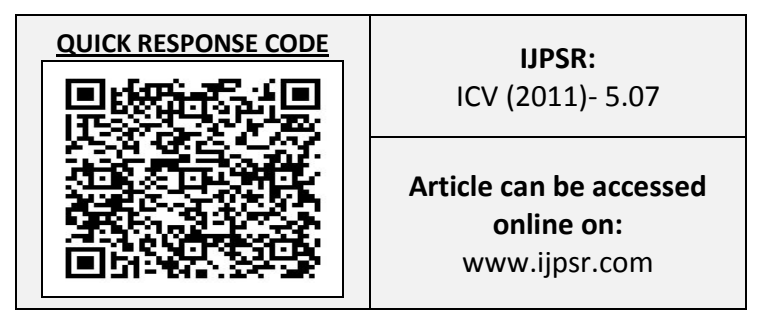

Diabetes mellitus with its accompanying complications such as cardiovascular diseases, retinopathy, nephropathy, and neuropathy is a serious health problem ${ }^{2}$. The complication risk is directly related to high blood glucose levels ${ }^{3}$. However, as most patients with type 2 diabetes mellitus have a combination of risk factors including obesity, hypertension, and hyperlipidemia ${ }^{4}$. The result of the Diabetes Control and Complication Trials ${ }^{5}$ and the United Kingdom Prospective Diabetes study 
demonstrated that the near- normalization of blood glucose level in patients with diabetes could significantly slow the progression of microvascular complications. Nevertheless, the risk of macrovascular complications did not reduce significantly. But some randomized clinical trials demonstrated the benefit of blood pressure lowering in diabetes, as reduction of coronary heart disease events, stroke, and nephropathy ${ }^{7}$.

The essential issues for managing of diabetes are patient's compliance to strict dietary, exercise, self care behavior, and medication regimens. Having concomitant disease, diabetes patients usually use polypharmacy, the greater the number of medications, the more drug related problems such as adverse drug reaction, drug interaction, medication non-compliance, no valid medical indication, and so on ${ }^{8}$. Poor medication compliance seems to be a significant barrier to the attainment of positive clinical outcomes among type 2 diabetes patients in both developed and developing countries ${ }^{9}$.

Increased pharmaceutical compliance was associated with fewer emergency department visit and patient admissions. Increased medication adherence was associated with decreased medical care cost ${ }^{10}$. In recent years, pharmacists in many practice settings have begun providing patient centered services with the goal of improving drug therapy outcomes through practices such as pharmaceutical care (PC). These PC programs have been found useful in improving the quality of care of patients with various diseases. Pharmacist's interventions in diabetes have also resulted in beneficial outcomes ${ }^{11}$.

The objective of this study was: To evaluate the efficiency of PC on the control of clinical parameters, such as FBS, glycosylated haemoglobin HbA1c in patients with type 2 diabetes mellitus and to reduce drug therapy problems among patients with T2DM.

\section{Methods:}

Study design: The study was a prospective, randomized, controlled trial. It was conducted at Diabetic Center in Sulaimani during 18 September 2010 to 20 January 2011.
Study population: The study populations were patients recruited by simple randomly sampling technique with the following criteria:

Inclusion criteria: Patients were included into to the study if:

1. Patients were diagnosed with type 2 diabetes mellitus.

2. Patients aged between 30 to 80 years old.

3. Having good orientation and ability to communicate verbally.

4. Agreeing to participate in the study.

Exclusion criteria: Patients were excluded from the study if:

1. Patients unable verbally to communicate: deaf, senile dementia, or Alzheimer's disease.

2. They were unable to undertake self-care or do not receive medications by themselves.

Study development: In this study, patients were divided into two groups by simple randomization technique; the first group is intervention group, who received pharmaceutical care, while the second one is control group who only received traditional medical care. The intervention group was followed up for 3 visits. The interval between each visit ranged from 5-6 weeks with continuous weekly telephone calling for the follow up.

Data Collection: The following data of each patient were recorded in the patient data collection forms.

General Demographic Data: (Name, file number, age, gender, weight, height, address, telephone number). Medical Data (Family history of diabetes, food or drug allergies, past medical history, past medication history, hypertension and hyperlipidemia).

Process evaluation data: Drug therapy problems and compliance by pill count and Morisky-Green test that consists of the following questions:

(1) Do you ever forget to take your medicine? 
(2) Are you careless at times about taking your medicine?

(3) When you feel better, do you sometimes stop taking your medicine?

(4) Sometimes if you feel worse when you take the medicine, do you stop taking it?

Clinical outcome data: Fasting plasma glucose (FPG), glycated hemoglobin (HbA1c)

Data analysis: Each returned questionnaire was given an identity number (ID). Prior to data entry and analysis, codes were inserted for each question. The data were entered into a Microsoft Excel
Spreadsheet, after data cleaning; the data were transported into SPSS (Statistical Package for the Social Sciences-verstion13.0) package software program for statistical analysis [12].Descriptive statistics (numbers and percentage) were calculated for all variables, as well as analytical statistics was done to find the relations between variables. Statistical test as Chi-square were used to find out the significant level. A p-value $<0.05$ was considered as significant.

RESULTS: Out of 130 patients, There were 123 patients who completed the study (62 patients in the intervention group and 61 patients in the control group). The other 7 patients were excluded because they came too late after first or second visits.

TABLE 1: THE DEMOGRAPHIC DATA OF BOTH GROUPS

\begin{tabular}{ccc}
\hline \multirow{2}{*}{ Variables } & \multicolumn{2}{c}{ Status } \\
\cline { 2 - 3 } Age groups & Control N (\%) & Case (Intervention) N (\%) \\
$30-49$ & $7(11.5)$ & $3(4.8)$ \\
$40-49$ & $15(24.6)$ & $23(37.1)$ \\
$50-59$ & $17(27.9)$ & $28(45.2)$ \\
$\geq 60$ & $22(36.0)$ & $8(12.9)$ \\
Mean \pm S.D. & $53.4 \pm 10.81$ & $52 \pm 7.86$ \\
\hline Sex & & \\
Female & $41(67.2)$ & $44(71.0)$ \\
Male & $20(32.8)$ & $18(29.0)$ \\
\hline $0-5$ & & \\
$6-10$ & $39(63.9)$ & $47(75.8)$ \\
$11-15$ & $18(29.5)$ & $10(16.1)$ \\
$16-20$ & $1(1.6)$ & $5(8.1)$ \\
Mean \pm S.D. & $3(4.9)$ & $0(0.0)$ \\
Durations of diabetes (Years) & $5.09 \pm 4.42$ & $4.12 \pm 3.42$ \\
\hline Negative & & $32(51.6)$ \\
Positive & $30(49.2)$ & $30(48.4)$ \\
\hline Hypertension & $31(50.8)$ & $35(56.5)$ \\
Normal & & $27(43.5)$ \\
Hypertensive & $32(52.5)$ & $29(46.8)$ \\
Hyperlipedimia & $29(47.5)$ & $33(53.2)$ \\
Normal & $37(60.7)$ &
\end{tabular}

At base line, FPG value were $211 \pm 70.3$ vs $249 \pm 88.9$ in the control and intervention groups, respectively and changes in FPG during the study in each group are shown in tables. The mean FPG shows a trend continuous improvement in the intervention group, but for the control group there is a fluctuation in the second visit, and at the end of the study the glycemic control and improvement of mean FPG were observed that reaches statistical significant differences in reduction in FPG for intervention group, but statistical significant differences in reduction in FPG for the control group were not obtained, as shown in Table 2. 
TABLE 2: FPG CHANGES DURING THE STUDY

\begin{tabular}{ccc}
\hline \multirow{2}{*}{ FPG readings } & \multicolumn{2}{c}{ Status } \\
\cline { 2 - 3 } FPG1 (mg/dl) & Control & intervention \\
Mean & 211.1 & 249.4 \\
SD & 70.3 & 88.9 \\
Range & $83-432$ & $95-485$ \\
\hline FPG2 (mg/dl) & & \\
Mean & 219.3 & 203.7 \\
SD & 57.77 & 84.3 \\
Range & $95-391$ & $83-459$ \\
\hline FPG3 (mg/dl) & & \\
Mean & 195.4 & 196.4 \\
SD & 62.6 & 74.2 \\
Range & $91-376$ & $93-361$ \\
\hline P- value & 0.196 & 0.001 \\
\hline
\end{tabular}

$\mathrm{P}$-value $<0.05$ considered as statistical significant differences

During this study, there are changes in the HbA1c value for both control and intervention groups, as summarized in table 3.

TABLE 3: HbA1c READINGS FOR BOTH GROUPS DURING EACH VISIT

HbA1C readings

Status

\begin{tabular}{ccc}
\cline { 2 - 3 } & Control & intervention \\
\hline HbA1Cl (\%) & & \\
Mean & 9.97 & 11.53 \\
Std. Deviation & 2.75 & 1.83 \\
Range & $8.6-14.5$ & $8.4-15$ \\
\hline HbA1Cll (\%) & & \\
Mean & 9.5 & 9.2 \\
Std. Deviation & 2.1 & 2.0 \\
Range & $5.2-15.6$ & $6.6-14.6$ \\
\hline P- value & $\mathbf{0 . 3 4 1}$ & $\mathbf{0 . 0 0 0}$ \\
\hline
\end{tabular}

$\mathrm{P}$ value less than 0.05 is significant

The mean HbA1c value at the end of the study shows tendency toward lower value than the first visit. This reduction in the $\mathrm{HbA} 1 \mathrm{c}$ reaches statistical significant differences; however, HbA1c value in the control group has a trend toward lower value from the baseline but was not statistically significant.

Medication Compliance: There are many methods for the assessment of medication compliance. In this study, indirect methods have been used (interview and pill count technique) for the evaluation of compliance among patients in the intervention group. Medication compliance from the first visit until the end of the study is summarized in Table 4.

TABLE 4: THE MEAN COMPLIANCE CHANGE AT EACH VISIT FOR INTERVENTION GROUP

\begin{tabular}{ccccc}
\hline \multirow{2}{*}{ Compliance change } & \multicolumn{3}{c}{ Visiting time } & \multirow{2}{*}{ P value } \\
\cline { 2 - 4 } & First visit N (\%) & Second visit N (\%) & Third visit N (\%) & \\
\hline Noncompliant & $48(77.4)$ & $26(41.9)$ & $12(19.0)$ & \multirow{2}{*}{$\mathbf{0 . 0 0 0}$} \\
Compliant & $14(22.6)$ & $36(58.1)$ & $50(81.0)$ & \\
\hline
\end{tabular}

There is an improvement in the medication compliance which is statistically significant (p value $<0.05$ ).

Drug Therapy Problems: Drug therapy problems (DTPs) were identified in the intervention group during the study. It is discussed in literature review (Table 16). DTPs are classified into 7 categories. In order to identify and resolve DTPs 7 points are considered among patients in the intervention group that include the followings:-

1. Unnecessary drug therapy.

2. Needs addition drug therapy.

3. Dosage too low.

4. Dosage too high.
5. Drug therapy ineffective.

6. Adverse drug reaction.

7. Noncompliance.

At the first visit, 23 patients have one DTP, 15 patients have two DTPs, and 3 patients have three DTPs, at the second visit two DTPs were identified among two patients and at the third visit just one DTP is found in one patient, considerable resolving and prevention of DTPs have been found that is statistically significant (P-value less than 0.05$)$.

TABLE 5: MEAN DTPS FOR THE INTERVENTION GROUP DURING THE STUDY

\begin{tabular}{|c|c|c|c|c|}
\hline \multirow{2}{*}{ DTP options } & \multicolumn{3}{|c|}{ Visiting time } & \multirow{2}{*}{ P value } \\
\hline & First visit $\mathbf{N}(\%)$ & Second visit N (\%) & Third visit $\mathbf{N}(\%)$ & \\
\hline No DTP & 21(33.9) & $59(95.2)$ & 61(98.4) & \multirow{4}{*}{0.000} \\
\hline One DTP & $23(37.1)$ & $3(4.8)$ & $1(1.6)$ & \\
\hline Two DTP & $15(24.2)$ & $0(0.0)$ & $0(0.0)$ & \\
\hline Three DTP & $3(4.8)$ & $0(0.0)$ & $0(0.0)$ & \\
\hline
\end{tabular}


TABLE 6: CHANGE IN THE MEAN DTPS SUBCATEGORIES FOR THE INTERVENTION GROUP DURING THE STUDY

\begin{tabular}{cccccccc}
\hline & $\begin{array}{c}\text { Unnecessary } \\
\text { NO. (\%) }\end{array}$ & $\begin{array}{c}\text { Needs } \\
\text { additional } \\
\text { NO. }(\%)\end{array}$ & $\begin{array}{c}\text { Ineffective } \\
\text { NO. }(\%)\end{array}$ & $\begin{array}{c}\text { Dosage too } \\
\text { low } \\
\text { NO. }(\%)\end{array}$ & $\begin{array}{c}\text { Adverse } \\
\text { reaction } \\
\text { NO. }(\%)\end{array}$ & $\begin{array}{c}\text { Dosage too } \\
\text { high } \\
\text { NO. }(\%)\end{array}$ & $\begin{array}{c}\text { Noncompliance } \\
\text { NO. (\%) }\end{array}$ \\
\hline $\begin{array}{c}1^{\text {st }} \\
\text { visit }\end{array}$ & $13(21 \%)$ & $13(21 \%)$ & $8(129 \%)$ & $31(50 \%)$ & $2(3.2 \%)$ & $5(8 \%)$ & $48(77.4 \%)$ \\
$2^{\text {nd }}$ & $0(0 \%)$ & $0(0 \%)$ & $0(0 \%)$ & $1(1.6 \%)$ & $2(3.2 \%)$ & $0(0 \%)$ & $26(41.1 \%)$ \\
$\begin{array}{c}\text { visit } \\
3^{\text {rd }} \\
\text { visit }\end{array}$ & $0(0 \%)$ & $0(0 \%)$ & $0(0 \%)$ & $0(0 \%)$ & $0(0 \%)$ & $1(1.6 \%)$ & $12(19.3 \%)$ \\
\hline
\end{tabular}

\section{DISCUSSION:}

Glycemic control: The data analysis showed there were significant reductions in HbA1c and FPG (9.19 $\pm 2.0 \%$ and $197.5 \pm 77.1 \mathrm{mg} / \mathrm{dl}$ respectively) obtained in the intervention group at the end of the study period. But there is no significant glycemic reduction in the control group. Our findings complied with those of other similar studies, Coast Senior et al ${ }^{13}$. The pharmacist's interventions in more recent studies also resulted in beneficial outcomes. In the study of Anaya et $a l^{14}$, Irons et al ${ }^{15}$ studied the quality of care of a pharmacist-managed diabetes clinic. Some other studies considered that pharmaceutical services did not result in an appreciable reduction in $\mathrm{HbA} 1 \mathrm{c}$ levels ${ }^{16,17}$.

At the end this study, glycemic level of $9(14.5 \%)$ patients did not change or very little changed, whereas, they received maximum therapeutic doses of antidiabetic medications. This inconsistent result could be explained by various reasons, for example: noncompliance to dietary control and exercise might explain this result. Chobchai et al ${ }^{18}$ reported that poor compliance to dietary recommendations, missed appointment, and medication noncompliance were found in $56.69 \%, 13.33 \%$ and $5.55 \%$ of patients, respectively.

A physically active lifestyle for individuals with diabetes reduces blood glucose levels and improves insulin sensitivity, inadequate regular exercise play an important role in the inadequate glycemic control. The American Diabetes Association (ADA) 2009 clinical practice recommendations state that "individuals who have pre-diabetes or diabetes should receive individualized $\mathrm{MNT}$ as needed to achieve treatment goals, the ADA's position statement titled "Nutrition Recommendations and Interventions for Diabetes" emphasizes the importance of MNT in preventing diabetes, managing existing diabetes, and preventing and slowing the onset of diabetes-related complications 19 . Huge numbers of the diabetic patients suffer from poor dietary control; these may suggest that diabetic patients may need dietary modification specialists to assist them.HbA1c and FBS results of intervention group were collected pre and post interventions and of control group during the three month period of study.

Demographic characteristics and laboratory test data of both groups were extracted from the patient's charts at the beginning and end of the three month period. The present study shows that better glycemic control was obtained in the intervention group and that the pharmacist education sessions, pill count, use of diary logs and follow up calls and appointments proved beneficial in reducing mean FBS and HbA1cs significantly. To our knowledge, this type of intervention is first of its kind to be reported in Iraqi Kurdistan region and shows that pharmacists could have added value in diabetes care management.

However, since an aggregate of techniques was utilized in the current intervention, it is difficult to show whether each individual component would have different impact on the final outcomes of the study. In the current study, patients were given advice for their glycemic control by a pharmacist every week; this continuous follow up may develop a trustable relationship between the pharmacist and patients. This close professional relationship as a part of this educational program might has contributed to a better diabetes control in the study.

Suppapitiporn et al revealed that consulting with the pharmacist in each visit improves the efficacy of glycemic control program ${ }^{20}$. Companions of patients during three months, close relationship existed between patients and pharmacist, acceptance of 
pharmacist's orders for diabetes control by patients and better glycemic control of patients in the intervention group show the acceptance of pharmacist by patients.

Medication compliance: Medication compliance was assessed in this study by direct methods, which include: Interview method and pill count method. In the first visit $48(77.4 \%)$ patients were noncompliant by interview (Morisky-Green test).

According to the protocol of the Morisky-Green test, patients are considered compliant to the treatment when they get a maximum score of four points, and noncompliant when they get three points or less ${ }^{21}$.

At the second visit, the number of noncompliant patients was reduced to $26(41.9 \%)$ patients by using Morisky-Green test and pill count technique. For the pill count technique, the percentage of each antidiabetic medication compliance was calculated for each patient; then the average percentage of all mediaction compliance was calculated, according to Sackett D.L if the result is more than or equal to $80 \%$ the patient is considered compliant ${ }^{22}$. At the third visit, compliance rate was assessed by MoriskyGreen test and pill count technique, the number of noncompliant patients was reduced to $12(19 \%)$ patients.

Our finding indicates that the major cause of noncompliance is forgetting to take medications (at the end of the study), the reasons of forgetting to take medications were mainly due to forgetfulness, being too busy, and/or hard working. These causes might be solved if patients learnt the importance of taking medicine at correct time and regularity. This study revealed that, the pharmacist may play an important role to teach patients about diabetic drugs and disease, to reduce the rate of noncompliance among diabetic patients, any efforts made by the pharmacist to share information with patients and engage them in talking about their medicines has the potential to improve compliance. However, patient's beliefs about their medicines are an invisible influence on their medicine-taking behavior, which cannot be addressed without discussion.

Drug Therapy Problems: Drug therapy problems (DTPs) were adapted from the definition of Cipolle and strand and it was classified into four categories: indication, efficacy, safety and noncompliance.
In this study, the noncompliance problems were the highest among DTPs. During the $2^{\text {nd }}$ and $3^{\text {rd }}$ visits for the intervention group, the intervention was accepted and the DTPs was solved in 91 cases (29 cases of DTP were not solved) and DTPs was solved in 16 cases (13 cases of DTP were not solved), respectively. One of the most common problems includes the time selected for the drug administration, for example: sulphonylureas should be administered 30 minutes before meal to control early postprandial plasma glucose strongly correlates with HbA1c levels and is associated with the development of microvascular and macrovascular diabetic complications. Taking sulphonylureas after meal may not decrease postprandial hyperglycemia and, consequently, HbA1c level may not be reduced.

\section{CONCLUSION:}

1. The study finding showed that there are significant reductions in glycemic levels for the intervention group this result suggested that, the implementation of the pharmaceutical care process could result in good glycemic control compared to usual medical care.

2. In term of medication compliance, this study showed that, pharmaceutical care process could increase the rate of medication compliance among patients in the intervention group.

3. Drug therapy problem is also one of the important issues for this study, the study findings showed that the numbers of patients who do not have any DTPs from the $1^{\text {st }}$ visit to the $3^{\text {rd }}$ are significantly increased. In addition, these findings contribute to the growing information in the literature that supports the development of clinical interventions and health policies in developing countries, stimulating the academic training of clinical pharmacists who are competent to act in healthcare teams.

It was concluded that diabetic patients were at risk of noncompliance to treatment and the incidence would be reduced if patients were intervened by the pharmacist. The study, therefore, suggested that the pharmaceutical care activities on the total care of the patients should be established in all chronic disease on a regular basis. 


\section{Recommendations for future works:}

1. On the basis of these results, clinical pharmacists should be integrated into healthcare team, to implement pharmaceutical care service for diabetic patients.

2. Other studies should be done to evaluate the impact of pharmaceutical care service for other chronic diseases. Compliance toward medications of chronic disease is an important issue to be assessed not only by pharmacists also by other healthcare teams.

3. Future research should focus on the quality of pharmaceutical care service in terms of economic outcome which includes cost consequences from detecting and resolving DTPs.

4. The sample size is relatively small; this study could be applied on a larger sample size.

\section{REFERENCES:}

1. International Diabetes Federation. Diabetes Atlas. 4th ed. Brussels; 2010; 100 p. 71-73

2. Matzer S, Leiter L, Daneman D, Gerstein H, Lau D, Ludwig S,et al. Clinical practice guidelines for the management of diabetes in Canada. CMAJ. 1998; 159:1-29.

3. American Diabetes Association: Implications of the diabetes control and complications trial. Diabetes Care 2002; 25: 2527.

5. Abuissa H, Bel DS, O'Keefe JH Jr. Strategies to prevent type 2 diabetes. Curr Med Res Opin. 2005; 21:1107-14.

6. The Diabetes Control and Complications Trial Research Group. The effect of intensive treatment of diabetes on the development and progression of long term complications in insulin- dependent diabetes mellitus. N Engl J Med 1993; (329):977-986.

7. United kingdom Prospective Diabetes Study Group. Intensive blood - glucose control with sulphonylureas or insulin compared with conventional treatment and risk of complications in patients with type 2 diabetes mellitus (UKPDS 33). Lancet 1998; (352): 837-853.

8. UK Prospective Diabetes Study Group. Tight blood pressure control and risk of macrovascular and microvascular complications in type 2 diabetes: UKPDS 38. BMJ. 1998; 317: 703-713.
9. Irons BK, Seifert CF, Horton NA. Quality of care of a pharmacist-managed diabetes service compared to usual care in an indigent clinic. Diabetes Technol Ther. 2008; 10:220 226.

10. Diehl AK, Bauer RL, Sugarek N. Correlates of medical compliance in non-insulin dependent diabetes mellitus. Diabetes Care. 1985; 8:219-223.

11. HepKe, K.L., Martus, M.T., \& Share, D.A. Costs and utilization associated with pharmaceutical adherence in a diabetic population. The AJMC ; 10, 144-151.

12. Brooks AD, Rihani RS, Derus CL. Pharmacist membership in a medical group's diabetes health management program. Am J Health Syst Pharm. 2007;64:617-621.

13. Appleton K, House A and Dowell A. A survey of job satisfaction, sources of stress and psychological symptoms among general practitioners in Leeds. British Journal of General 1060 Practice. Br J Gen Pract. 1998; 48: 1059-1063.

14. Coast-Senior EA, Kroner BA, Kelley CL, Trilli LE. Management of patient with type 2 diabetes by pharmacists in primary care clinics. Ann Pharmacother. 1998; 32:636-41.

15. Anaya JP, Rivera JO, Lawson K, Garcia J, Luna J Jr, Ortiz M. Evaluation of pharmacist-managed diabetes mellitus under a collaborative drug therapy agreement. Am J Health Syst Pharm. 2008;65:1841-5.

16. Irons BK, Seifert CF, Horton NA. Quality of care of a pharmacist- managed diabetes service compared to usual care in an indigent clinic. Diabetes Technol Ther. 2008; 10:220-6.

17. Rothman R, Malone R, Bryant B, Horlen C, Pignone M. Pharmacist led, primary care-based disease management improves hemoglobin HbA1c in high-risk patients with diabetes. Am J Med Qual. 2003;18(2):51-8.

18. Irons BK, Lenz RJ, Anderson SL, Wharton B, Habeger B, Anderson G. A retrospective cohort analysis of the clinical effectiveness of a physician-pharmacist collaborative drug therapy management diabetes clinic. Pharmacotherapy. 2002; 22(10): 1294-300.

19. Chobchai p. survey of dietary supplements and herbs use in type2 DM, banking university. 2006; P. 2545-2557.

20. American Diabetes Association: Executive summary: standards of medical care in diabetes. Diabetes Care2009; 32:S6-S12.

21. Suppapitiporn S, Chindavijak B, Onsanit S. Effect of diabetes drug counseling by pharmacist, diabetic disease booklet nd special medication containers on glycemic control of type 2 diabetes mellitus: a randomized controlled trial. Med Assoc Thai 2005;88 Suppl 4:S134-41.

22. Morisky DE, Green LW, Levine DM. Concurrent and predictive validity of self-reported measure of medication adherence. Med Care. 1986; 24(1):67-74.

23. Sackett DL: Hypertension in the Real World: Public Reaction, Physician Response and Patient Compliance. In: Genest J.. Kevin E., Kechal $\mathrm{P}$ eds. Hypertension: Pathophysiology and Treatment, New York: McGraw-Hill; 1977. 1142.

\section{How to cite this article:}

Mahwi TO and Obied KA: Role of the Pharmaceutical care in the management of patients with Type 2 Diabetes mellitus. Int $J$ Pharm Sci Res 2013; 4(4); 1363-1369. 